\title{
Imagery, cerebral dominance, and style of thinking: A unified field model
}

\author{
ROBERT ZENHAUSERN \\ St. John's University, Jamaica, New York 11439
}

\begin{abstract}
A model of thinking style, based on whether a person thinks in pictures or does not think in pictures, was proposed. Right cerebral dominance and a deductive mode of reasoning were associated with the visualizing style, while left cerebral dominance and an inductive mode of reasoning were associated with the nonvisualizing style. Converging lines of evidence (anecdotal, historical, and empirical) were offered in support of the model.
\end{abstract}

The major controversy in imagery research is best exemplified by the polar positions of Paivio (1971) and Pylyshyn (1973). The dual coding model of Paivio stresses the value of imagery as a second encoding system, along with verbal encoding, the two working together in memory tasks. Pylyshyn, on the other hand, considers imagery as a concomitant of thought processes and not important in those processes.

The model proposed in this paper would consider the positions of Paivio and Pylyshyn to be, at least in part, a function of their own thinking styles. That is, some people always think in images and either cannot think without them or can do so only with difficulty. Other people have either no imagery at all when they think, or have imagery that is merely an accompaniment to their thinking, and of little importance. Consider a person who is left cerebral dominant. ${ }^{1}$ The left hemisphere inputs information sequentially, analyzing and storing that information. Retrieval of that information is also sequential, leading to a synthetic or inductive style of reasoning. This process is equivalent to recalling the stored propositions of Pylyshyn (1973) and other investigators and building a conclusion from them: an inductive process. It is interesting to note that, while the left hemisphere is analytic in input and retrieval, it is synthetic in processing.

Now consider an individual who is right cerebral dominant. The right hemisphere inputs information holistically, synthesizing the information. It is unclear how the information is stored, in propositional or picture form. Anderson (1978) has concluded that, given our present state of knowledge, it is not possible to distinguish between the two storage models. In whatever form the information is stored, it is retrieved holistically. If the information is retrieved synthetically and in parallel, an image would seem to be the most economical form of retrieval. Given this image, the various aspects can then be analyzed and examined individually: a deductive process. Thus, the right hemisphere inputs and retrieves synthetically but processes analytically.

In summary, this model states that individuals think in one of two ways: in pictures or not in pictures. Those who think in pictures either cannot think without them or can do so only with difficulty, while those who do not think in pictures may have images accompaying their thoughts, but they are not essential and may be eliminated. The nature of the thought processes of those who do not think in pictures is unclear. It is sometimes described as "similar to hearing" or "it's just there." Since those who think in pictures can be very verbal, the verbalizer-visualizer dimension of A. Richardson (1977) can lead to confusion. Thus, the present model proposes a visual-nonvisual dichotomy. There is some evidence that a small number of people can think in either mode, and this may be equivalent to mixed cerebral dominance. A dichotomy has been proposed at this early stage because of its simplicity. Future work may indeed indicate that a person thinks predominately in one or the other of these modes. In this case, the predominate mode of thought would serve as a classifying variable.

\section{SOME IMPLICATIONS OF THE MODEL}

The controversy exemplified by the positions of Paivio (1971) and Pylyshyn (1973) is less a controversy than a reflection of their own (presumed) thinking styles. The nonvisualizer processes information without imagery, or with imagery as an epiphenomenon, not essential to his thinking processes. The dual coding model is correct except that both coding procedures probably do not coexist within the same individual.

Recently, J. Richardson (1978) pointed out the lack of consistent results when imagery is treated as a classifying variable, either through self-report or through performance measures of imagery. This is easily explained by this model in terms of classification according to the wrong variable. Nonvisual thinkers may have rather vivid imagery accompanying their thinking, and these individuals are difficult to differentiate from visual thinkers on introspective reports. The use of objective tests, such as "flags," may not solve the problem either, since nonvisual thinkers may 
be able to obtain relatively high scores through some form of verbal mediation. J. Richardson (1978) concludes that visual or nonvisual coding preference, rather than coding ability as measured by subjective or objective imagery tests, should serve as the classifying variable. This is the essence of the present model.

\section{EVIDENCE IN SUPPORT OF THE MODEL}

Three converging lines of evidence supporting the model will be considered in the next section: anecdotal, historical, and empirical. No direct experimental testing of the model has been completed, but research in the areas of verbal learning, sense of direction, inductive and deductive reasoning, and cerebral dominance are in progress.

\section{Anecdotal Evidence}

Since subjective and objective tests of imagery ability have not resulted in predicted performance differences (see, especially, J. Richardson, 1978), the only way to determine if a person thinks visually or nonvisually is to ask that question directly. People have been able to make this distinction quite well if the right questions are asked. Visual thinkers are usually very definite in their conclusions that they do think in pictures. One 16-year-old boy, when asked how he thought, responded, "Do you mean, how do I picture things?" Nonvisual thinkers, on the other hand, have more problems because, not only may they have images accompanying their thoughts, but also, it is very difficult to describe exactly what actually occurs.

One important finding is that many nonvisual thinkers have rather vivid imagery, but they can state with confidence that they do not think in pictures. One nonvisual thinker who had extremely vivid imagery even refused to believe that it was possible for anyone to think in pictures. A questionnaire, now in its third revision, has been successful in differentiating the two thinking styles, especially with the inclusion of two questions that ask the subject to try to think without using pictures. These questions seem to be able to separate visualizers from nonvisualizers with good imagery.

Since the questionnaire requires a person to classify himself as a visual or nonvisual thinker, what appears to be a dichotomy may, on more precise measurement, be modified to a predominately visual or nonvisual thinking style. This would be consistent with the fact that different problems demand different cognitive solutions. On the other hand, subjects do seem to be comfortable with the dichotomy approach, except for three people who reported that they used both thinking sytles, depending on the situation. The latter group may be reflective of ambidominant individuals. The evidence from subjective reports, therefore, supports the dual style-of-thinking aspect of the model.

\section{Historical Evidence}

The major assumption of this section is that people, when they theorize about thinking, will base their formulations on the thinking with which they are most familiar: their own. Price (1953) discusses two major theories of thinking, imagism and nominalism, which correspond to the visual and nonvisual modes proposed by the present model. The disagreement between Berkeley and Locke as to whether it is possible to have an abstract thought can be seen as a reflection of their personal styles of thinking. Berkeley was a visualizer (Armstrong, 1965; Price, 1953) and, given that he thought in images, it is easy to see how he considered it impossible to consider "whiteness," "extension," "motion," and so on, separately from objects that exhibit those characteristics. Furthermore, Berkeley (cited in Boring, 1957) argued that the mind generates matter. The entire world, he argued, is contained within the mind, and specific facts flow from the mind: a deductive process. Locke, on the other hand, was a nonvisualizer (Price, 1953). Since he did not think in images, the same abstractions that Berkeley denied formed the basis of his thinking. In addition, he wrote that the world generates the mind. People are born with a tabula rasa, and the mind is formed by perceiving the world. A simple idea enters the mind through the senses and these simple ideas are compounded into complex ideas. This is obviously an inductive process. Thus Locke was a nonvisualizer and an inductive thinker, while Berkeley was a visualizer and a deductive thinker.

Further historical evidence is seen in the imageless thought controversy that raged during the turn of the century (see Boring, 1957). Wundt and his followers denied the possibility of imageless thought. Immetliate experience was the proper study of psychology, and experience was analyzed into specific components: a deductive approach. The Wurzburg school, on the other hand, postulated imageless thought and studied the mind by asking subjects to describe their thought processes. From these specific introspective reports, it was hoped that the entire thought process could be determined: an inductive approach.

Roe (1950) investigated the thought processes of research scientists and found three major groupings: those who thought in visual images, in verbal images, and without imagery. In the present model, the latter two groups are combined into a nonvisualizer group. It is difficult for subjects to make such a distinction, and it is not relevant to the model in its present form. This may change with future refinements of measurement and research aimed at this distinction.

The historical evidence just cited lends support to the idea that visual thinking is associated with deductive reasoning and nonvisual thinking is associated with inductive reasoning. Since cerebral dominance is a relatively new concept, the evidence linking it to visual 
and nonvisual thinking will be considered in the next section on recent empirical studies.

\section{Empirical Evidence}

The purpose of this section is to present the evidence that imagery is associated with the right hemisphere and especially with right hemisphere dominance. Galin and Ornstein (1974) found that subjects involved in imagery tasks have higher alpha amplitude in the left hemisphere compared to the right. Since alpha amplitude is indicative of relative idling in processing, this finding is indicative of a right hemisphere specialization in imagery. Corsi (cited in Cohen, 1977) found that patients with right hemisphere dämage were đeficient in picture recognition, but performed normally on verbal tasks. Carrammazza, Gordon, Zurif, and DeLuca (1976), working with subjects with right hemisphere damage, concluded that these subjects failed on certain tasks because visualization was essential to completion of the task and the damaged hemisphere was incapable of this process.

A. Richardson (1978) reported that male subjects classified as visualizers on the visualizer-verbalizer dimension had more left eye movements to a series of 20 questions than subjects who were classified as verbalizers. For female subjects, the sex and style (verbalizer-visualizer) of the examiner was an important variable. Since left eye movements are associated with right hemisphere activity (Kimura, 1973; Nebes, 1974), this is indicative of greater right hemisphere involvement for the visualizer than for the verbalizer. The complex results found for females may reflect the typically reported less complete lateralization for females or the inadequacy of the verbalizer-visualizer dimension as a categorical variable.

J. Richardson (1978), using subjects with high and low scores on a subjective imagery questionnaire and on a performance measure of imagery, developed four groups of subjects (high on both tests, low on both tests, and high on one and low on the other). In three experiments investigating free recall, coding ability, and coding preference involving verbal and pictorial stimuli, as well as high- and low-imagery words, there was no evidence of differential performance for the four groups. These nonsignificant differences are indicative of the inadequacy of subjective and objective tests of imagery to consistently separate the cognitive styles of individuals classified as visualizers and nonvisualizers in the present model. That is, imagery ability cuts across thinking style and, as J. Richardson puts it, coding preference may be the critical variable.

Gur and Hilgard (1975) separated subjects on subjective imagery ability and measured the time to judge two pictures to be the same or different, using both simultaneous and successive (20-sec delay) presentations.
The difference between successive and simultaneous presentations was not significant for the good imagers, but successive presentation took significantly more time than simultaneous presentation for the poor imagers. The introspective reports of the subjects are particularly relevant to this paper. "The difference in reaction time between 'good' and 'poor' imagers suggests, however, that they used the information in different ways. The 'poor' imagers recalled details of the original picture, then searched the new picture for difference. ... The 'good' imagers . . . commonly reported that the difference 'popped out,' as though the original picture was a matrix that could be placed over the second one for the detection of the difference" (Gur \& Hilgard, 1975, p. 344).

The poor imagers obviously processed the material in the sequential style indicative of the left hemisphere and in what is clearly an inductive mode of processing. The good imagers, on the other hand, operated in the holistic style of the right hemisphere, using a deductive mode of processing. A comparison of the negative results of J. Richardson (1978) and the positive results of Gur and Hilgard (1975) points up the inconsistent findings that occur when subjective or objective tests of imagery are used to classify subjects. It is proposed that these inconsistencies would disappear if the visualizernonvisualizer dichotomy proposed in the present model were used in the differentiation of subjects.

\section{Preliminary Questionnaire Results}

A questionnaire ${ }^{2}$ measuring styles of thinking has been administered to 66 graduate and undergraduate students, along with tests of subjective and objective imagery and another test of thinking style. A complete analysis of these results will be available in the near future. Thirty-five subjects indicated that they thought in pictures; 20 of these reported very vivid imagery. Of the 31 subjects who felt they did not think in pictures, only 7 reported very vivid imagery, although 10 indicated moderate imagery. Only three subjects said they had no imagery at all. An additional three subjects could not make the distinction regarding the way they thought. From these findings, two conclusions may be drawn: (1) Subjects are able to report whether they think in a visual or a nonvisual mode, and (2) imagery ability cuts across the visualizer-nonvisualizer categories, indicating that imagery is not a reliable classifying variable.

In summary, the traditional methods of differentiating subjects on the basis of imagery ability have yielded inconsistent results because the appropriate classifying variable is not objective or subjective imagery ability, but rather visual or nonvisual thinking style. Furthermore, these two thinking styles are a direct consequence of cerebral dominance and lead to deductive or inductive processing modes. 


\section{REFERENCES}

ANDERSON, J. Arguments concerning representations for mental imagery. Psychological Review, 1978, 85, 249-277.

ARMSTRONG, D. (Ed.). Berkeley's philosophical writings. New York: Collier, 1965.

Boring, E. A history of experimental psychology. New York: Appleton-Century-Crofts, 1957.

Carrammazza, A., Gordon, J., Zurif, E., \& Deluca, D. Right hemisphere damage and verbal problem solving behavior. Brain and Language, 1976, 3, 41-46.

Conen, G. The psychology of cognition. New York: Academic Press, 1977.

Galin, D., \& Ornstein, R. Individual differences in cognitive style. I. Reflective eye movements. Neuropsychologia, $1974,12.367-390$.

Gur, R.. \& Hilgard, E. Visual imagery and the discrimination of differences between altered pictures simultaneously and successively presented. British Journal of Psychology, 1975, 66, 341-345.

Kimura, D. The asymmetry of the human brain. Scientific American, 1973, 228, 70-77.

NeBES, R. Hemispheric specialization in commisuratomized man. Psychological Bulletin, 1974, 81, 1-4.

Paivio, A. Imagery and verbal processes. New York: Holt, Rinehart, \& Winston, 1971.

Price, H. Thinking and experience. Cambridge: Harvard University Press, 1953.
Pylyshyn. Z. What the mind's eye tells the mind: A critique of mental imagery. Psychological Bulletin. 1973, 80, 1-24.

Richardson, A. Verbalizer-visualizers: A cognitive dimension. Journal of Mental Imagery. 1977. 1. 109-126.

Richardson. A. Subject, task, end tester variables associated with initial eye movements. Journal of Mental Imagery. 1978, 2, 85-100.

Richardson, J. Mental imagery and memory: Coding ability or coding preference? Journal of Mental Imagery, 1978, 2. 101-116.

RoE, A. A study of imagery in research scientists. Journal of Personality, 1950, 19, 459-470.

\section{NOTES}

1. The term cerebral dominance is not clearly defined in the literature. For the purpose of the present model, the following operational definition is used. If a subject performing a lateralized task is asked to process verbal information in the left hemisphere and pictorial information in the right hemisphere, a left-dominant individual will be more efficient on the former than on the latter, while the opposite will be true for a rightdominant individual.

2. Copies of the questionnaire are available from the author.

(Received for publication August 7, 1978.) 